\title{
LA INFLACIÓN DEL ECUADOR
}

Desde la antigüedad la inflación es uno de los mayores problemas que aquejan al mundo en general, en especial a gobiernos débiles, países tercermundistas donde se refleja que mientras más frágil es el gobierno más alta será su inflación; el inconveniente se presenta porque cada vez se disminuye el poder adquisitivo de la población, ya que los gastos no se reducen sino se incrementan, debido al alza generalizada de precios teniendo que disminuir su capacidad de compras con los mismos salarios, lo que ocasiona un deterioro en el nivel de vida de la población, esto demuestra que la inflación es un distorsionador del orden natural de precios lo que impide una correcta asignación de recursos.

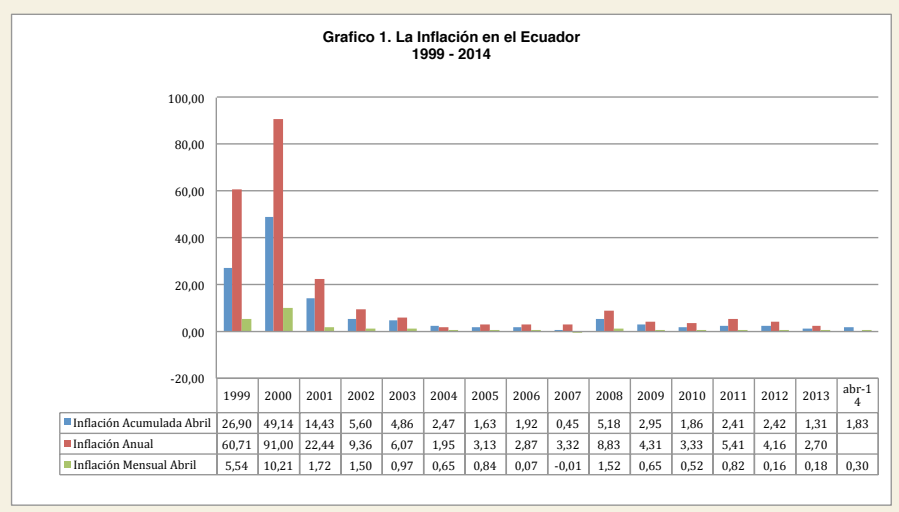

Fuente: Instituto Nacional de Estadísticas y Censos

Elaboración: Equipo Técnico del Observatorio Económico y Social de Tungurahua

En el grafico 1, se puede observar que la economía del Ecuador entró en una terrible crisis económica en el Gobierno de Jamil Mahuad en la recesión de 1999 causado por la crisis bancaria, la alta devaluación anual, debido a la emisión inorgánica deficiente que realizó el Banco Central a inicios del año, disminución en el precio del petróleo, todos estos factores provocaron una inflación anual de $60.71 \%$, posteriormente se dio paso a un feriado bancario y después la incautación de los depósitos en sucres para salvar a la banca y de esta forma disminuir las presiones especulativas sobre la divisa.
El 9 de enero del 2000 se implementa la dolarización con una conversión de 25000 sucres por cada dólar, se adopta este esquema con el propósito de bajar la inflación y estabilizar la economía ecuatoriana.

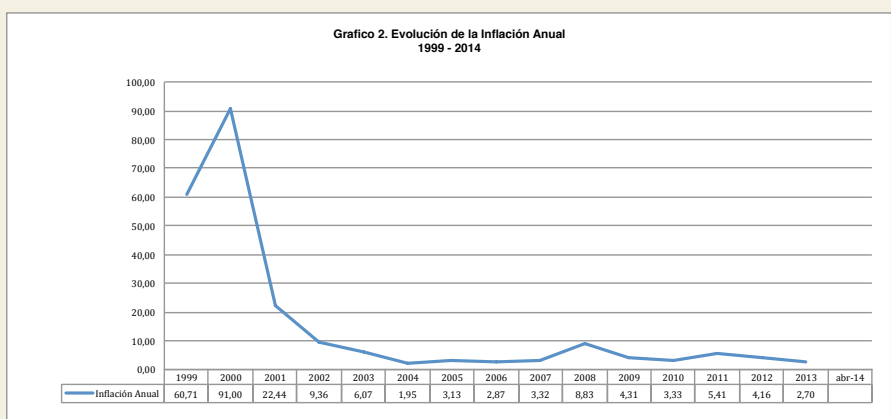

Fuente: Instituto Nacional de Estadísticas y Censos

Elaboración: Equipo Técnico del Observatorio Económico y Social de Tungurahua

En el Grafico 2, se puede observar que a partir del 2001 se empieza a estabilizar la economía y la inflación se disminuye del 2000 al 2001, en el año 2002 alcanzó el 9,36 \% en el 2003 la tendencia sigue a la baja.

Durante el primer año de gobierno del Economista Rafael Correa en el 2007 terminó con una inflación de 3,32\% con un incremento respecto al año 2006 y para el 2013 cerró con 2,70\% siendo éste el índice inflacionario más bajo durante sus gobiernos.








Fuente: Instituto Nacional de Estadísticas y Censos

Elaboración: Equipo Técnico del Observatorio Económico y Social de Tungurahua

En el gráfico 3, se puede determinar que la inflación acumulada más alta en el 2006, fue en la ciudad de Machala con 2,75\% y la más baja en Loja de 0,89\%, en el 2007 Manta con 1,12\% mientras que en Quito existe una deflación del 0,17\% lo que se pudo causar por una caída en el consumo o a un exceso de oferta, en el 2008 nuevamente Esmeraldas con 7,89\% y Cuenca con $3,12 \%$; en el 2009 se destaca que Manta es la ciudad con mayor porcentaje con 4,25\%; en el 2010 hay un nivel casi similar entre las ciudades, siendo Loja la ciudad con menor índice.

En el 2011 Ambato con 3,26\%; en el 2012 Manta con 3,46\%; en el 2013 Machala con 2,56\% y finalmente en el 2014 Esmeraldas y Ambato con 2,75\%.

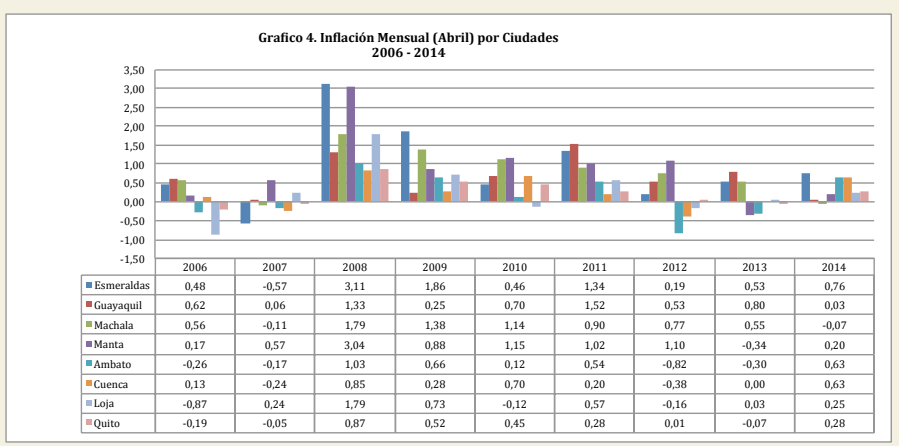

Fuente: Instituto Nacional de Estadísticas y Censos

Elaboración: Equipo Técnico del Observatorio Económico y Social de Tungurahua
En el gráfico 4 de la inflación mensual de abril por ciudades se puede determinar que durante este periodo Esmeraldas, Guayaquil y Manta fueron las ciudades con mayor inflación durante tres años y con niveles de baja inflación y deflación fueron Cuenca en 3 años, seguida de Quito en 2 años.

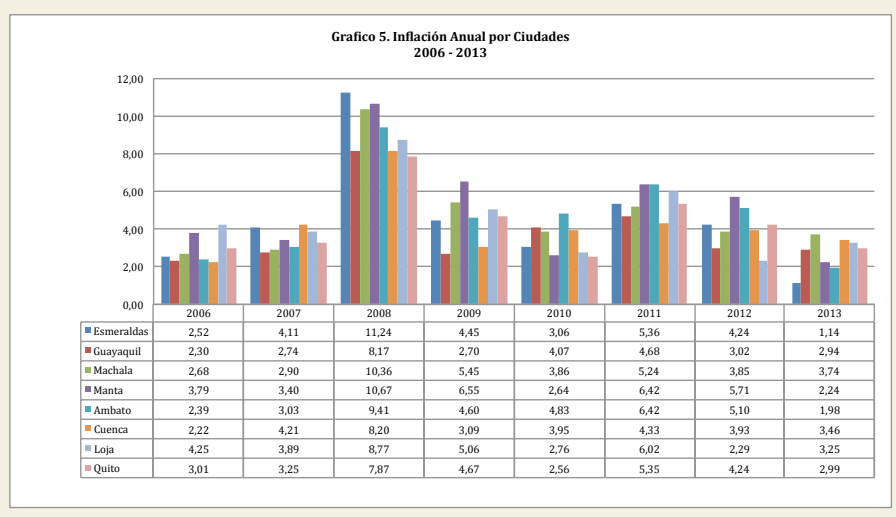

Fuente: Instituto Nacional de Estadísticas y Censos

Elaboración: Equipo Técnico del Observatorio Económico y Social de Tungurahua

En el gráfico 5, se encuentran las inflaciones anuales del 2006 al 2013, siendo Esmeraldas en el 2008 la ciudad con mayor índice inflacionario con 11,24\% seguido de Machala con 10,36\%; y, el año con menor inflación fue el 2013 en Esmeraldas con 1,14\% seguido de Ambato con 1,98\%.

Tabla 1. Canasta Básica y Vital del Ecuador 2006 - 2014

\begin{tabular}{|c|c|c|c|c|}
\hline Años & $\begin{array}{l}\text { Canasta } \\
\text { Básica } \\
\text { USD }\end{array}$ & $\begin{array}{c}\text { Canasta Vital } \\
\text { USD }\end{array}$ & $\begin{array}{l}\text { Ingreso } \\
\text { Familiar } \\
\text { USD }\end{array}$ & $\begin{array}{l}\text { Propensión (+) o } \\
\text { Restricción (-) en } \\
\text { el consumo USD }\end{array}$ \\
\hline 2006 & 453,26 & 306,56 & 298,67 & $-154,59$ \\
\hline 2007 & 472,74 & 330,39 & 317,34 & $-155,40$ \\
\hline 2008 & 508,94 & 358,83 & 373,34 & $-135,60$ \\
\hline 2009 & 528,90 & 377,87 & 406,93 & $-121,97$ \\
\hline 2010 & 544,71 & 390,10 & 448,00 & $-96,71$ \\
\hline 2011 & 578,04 & 419,25 & 492,80 & $-85,24$ \\
\hline 2012 & 595,71 & 431,32 & 545,07 & $-50,64$ \\
\hline 2013 & 620,86 & 444,78 & 593,60 & $-27,26$ \\
\hline$a b r-14$ & 633,61 & 453,66 & 634,67 & 1,06 \\
\hline
\end{tabular}

Fuente: Instituto Nacional de Estadística y Censos

Elaboración: Equipo Técnico del Observatorio Económico y Social de Tungurahua 
En la tabla 1, encontramos datos de las canastas básica, vital y de ingresos, por lo que se debe diferenciar que es una de ellas. Se determina según la metodología del INEC que la canasta básica posee 75 productos que se usan para controlar el incremento de precios y a relacionarlos con las remuneraciones de la población, su capacidad de consumo, en cambio la canasta vital, que es un conjunto de 73 artículos, en menor cantidad y calidad que la canasta básica, y se la llama vital porque señala el límite mínimo de supervivencia de una familia. La diferencia radica en que están determinadas por la cantidad y calidad de los productos que las componen.

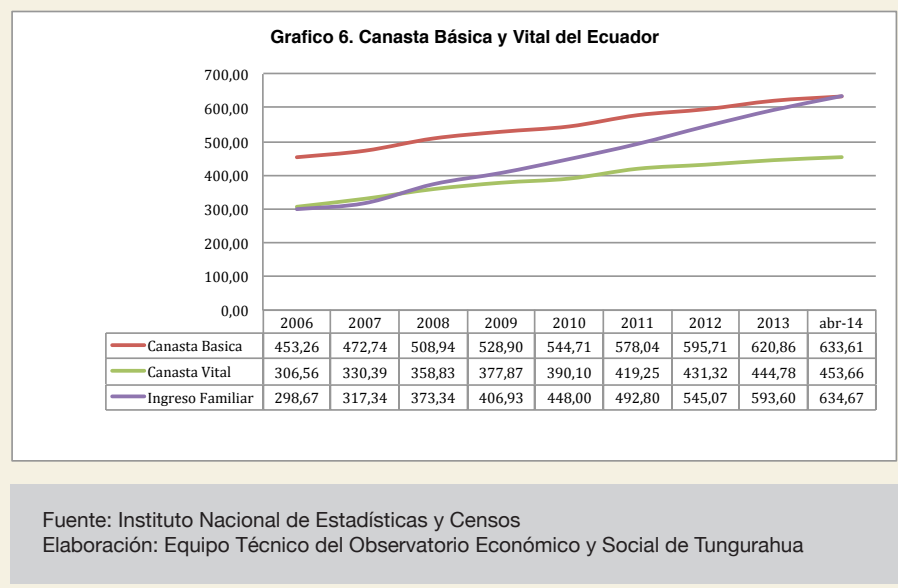

En el Gráfico 6, se puede determinar que existe un incremento de la Canasta Básica Familiar y la Canasta Vital del 36,98\% y $45,09 \%$ respectivamente, entre los años 2006 y 2013; sin embargo, durante el mismo período los ingresos familiares crecieron en un $98,75 \%$. La Canasta Básica para el mes de abril de 2014 fue de 633,61 dólares, la Canasta Vital 453,66 dólares y el ingreso familiar 634,67 dólares.

El aumento del ingreso familiar reduce la brecha entre la canasta básica y vital, para de esta forma se incremente el poder adquisitivo de las familias de nuestro país. Se puede determinar que el ingreso fue mayor a la canasta vital a partir del año 2008 al 2011, además durante este periodo se establece el salario digno que busca que el salario llegue a cubrir por lo menos la canasta básica.

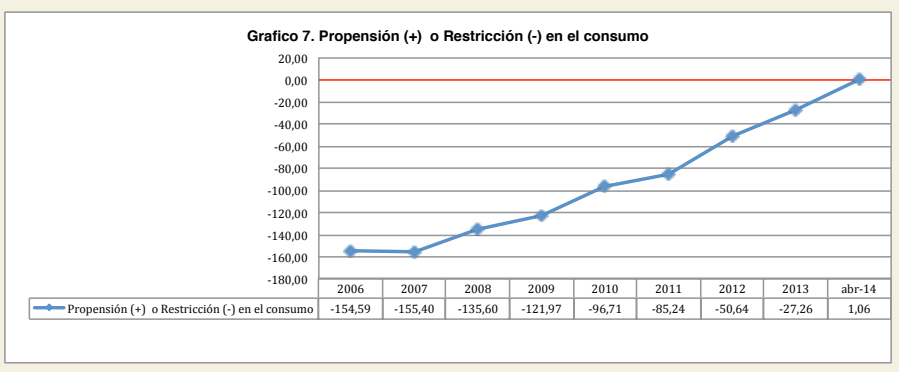

Fuente: Instituto Nacional de Estadística y Censos

Elaboración: Equipo Técnico del Observatorio Económico y Social de Tungurahua
En el gráfico 7 se determina que, en el transcurso de los años las familias no han logrado cubrir con sus ingresos la canasta básica lo que ha provocado restricción en el consumo reduciendo la calidad de vida de los ecuatorianos durante el periodo analizado (2006 - 2013), siendo el 2007 año en el que su poder adquisitivo sufrió mayores estragos con 155,40 dólares, pero cabe señalar que a través de los años se ha reducido favorablemente el valor de restricción para destacar que en el 2013 fueron ya 27,26 dólares, marcando una gran diferencia respecto al año 2007.

Lo que se determina para este año es aun mejor el panorama, porque en los primeros meses se ha podido detectar una propensión al consumo y en el mes de abril de este año se ha logrado tener un valor de 5,11 dólares adicionales al valor de la canasta básica.

El valor de la canasta familiar básica se ubicó en 633,61 dólares, mientras que el ingreso familiar en 634,67 dólares, esto implica una cobertura del $100,17 \%$ del costo de dicha canasta, con un valor a favor para el consumo de 1,06 dólares y un superávit del $0,17 \%$.

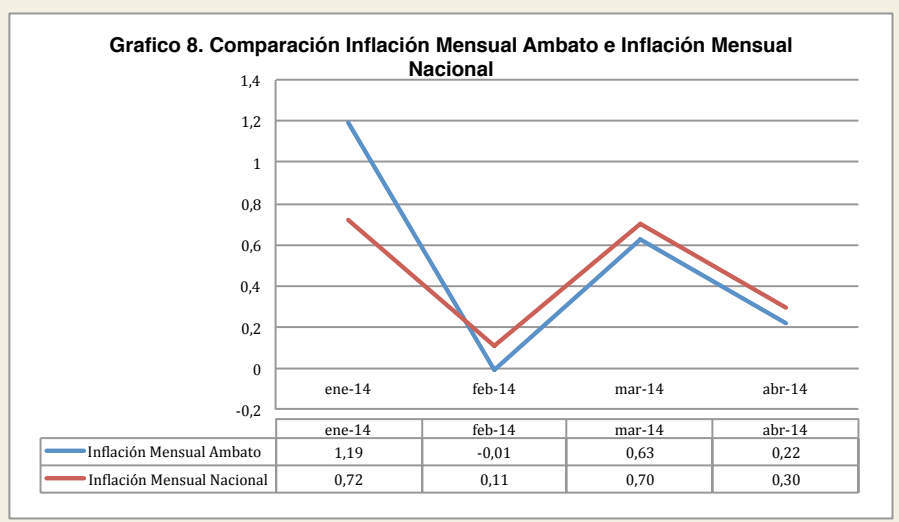

Fuente: Instituto Nacional de Estadística y Censos

Elaboración: Equipo Técnico del Observatorio Económico y Social de Tungurahua

En el grafico 8, se puede observar el ritmo que ha tenido la inflación durante los cuatro primeros meses del 2014 en la ciudad de Ambato con respecto a la inflación mensual y Nacional, donde se puede determinar que a inicios de año en el mes de enero superó en $0,47 \%$ la inflación de Ambato con respecto a la Nacional, pero a partir del mes de febrero hasta el mes de abril siempre ha sido inferior la inflación mensual de esta ciudad con respecto a la Nacional. 




Fuente: Instituto Nacional de Estadística y Censos

Elaboración: Equipo Técnico del Observatorio Económico y Social de Tungurahua

En el gráfico 9, el valor de la canasta familiar básica de los ambateños durante los cuatro primeros meses se ha incrementado en un $1,78 \%$ es decir 10,57 dólares de enero hasta abril de este año, la canasta vital de igual manera ha incrementado en un $3,54 \%$, es decir 15,06 dólares.

Gráfico 10. Propensión en el consumo en Ambato Enero - Abril 2014 USD

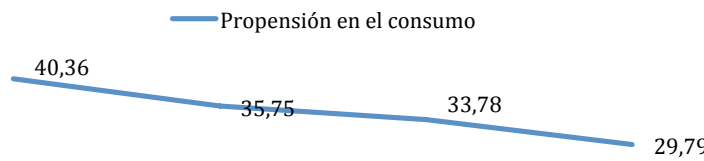

ene-14

feb-14

$\operatorname{mar}-14$

abr-14

Fuente: Instituto Nacional de Estadística y Censos

Fuente: Instituto Nacional de Estadística y Censos
Elaboración: Equipo Técnico del Observatorio Económico y Social de Tungurahua
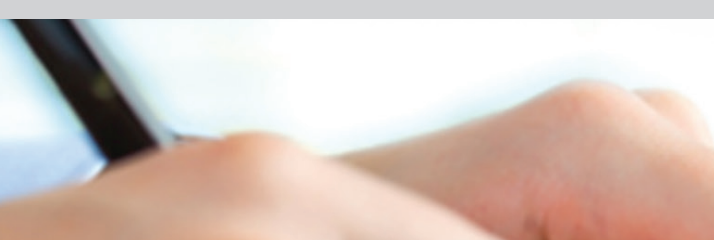

Algo que se puede detectar con el gráfico 10, es que los ingresos de los ambateños están cubriendo la canasta básica de esta población, ya que existe una propensión al consumo aumentando el poder adquisitivo de los ambateños.

\section{Referencias}

- Instituto Nacional de Estadísticas y Censos, Reporte y Presentación de Inflación (En Línea), disponible en: http://www. ecuadorencifras.gob.ec/ecuador-registra-una-inflacion-de030-en-abril/

- Instituto Nacional de Estadísticas y Censos, Canasta Familiar (En Línea), disponible en: http://www.ecuadorencifras. gob.ec/ipc-canastas-2014/

- Agencia Pública de Noticias del Ecuador y Suramérica, Andes, (En Línea).

- Zona Económica, (En Línea).

Observatorio Económico y Social de Tungurahua (OBEST) Universidad Técnica de Ambato.

\section{Econ. Tatiana Vayas}

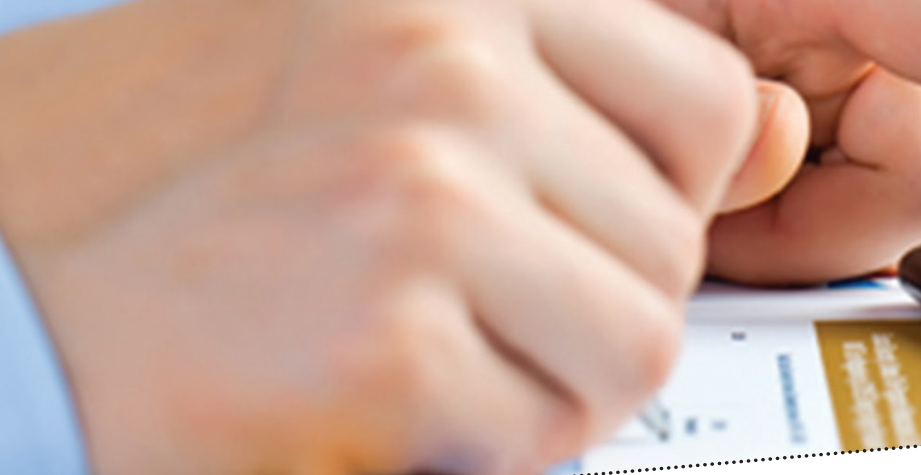

